\title{
FIZZ1 potentiates the carbachol-induced tracheal smooth muscle contraction
}

\author{
H. Chen, B.A. Jacobson, L. Mason, S.F. Wolf and M.R. Bowman
}

ABSTRACT: FIZZ1 is an adipokine highly expressed under inflammatory conditions, and yet, little is known of its function. In this study we examine the expression and function of $\mathrm{FIZZ1}$ in an ovalbumin mouse model of asthma.

Trachea from naïve or ovalbumin-sensitised and -challenged mice were compared for transcriptional, functional and proteomic differences using gene microarrays, ex vivo tracheal contraction, immunohistochemistry and Western blot analysis.

FIZZ1 was expressed in ovalbumin-treated, but not naïve, trachea. Naïve trachea incubated with recombinant FIZZ1 exhibited denuded epithelium and contractile hyperresponsiveness. The FIZZ1-incubated trachea also exhibited an associated increased expression of phospho-c-Raf, phospho-extracellular signal-regulated kinase 1/2, phospho-p38, MLCK and MLC-20.

These data demonstrate that FIZZ1 regulates tracheal smooth muscle contraction through impairment of the epithelium and activation of the mitogen-activated protein kinase pathway in muscle.

KEYWORDS: Airway epithelium, c-Raf, extracellular signal-regulated kinase 1/2, mitogenactivated protein kinase, myosin light chain kinase, myosin light chain 20

IZZ1, Resistin-like molecule- $\alpha$, is a secreted protein of the resistin family of adipokines. It is released from a variety of cell types including airway epithelial cells. FIZZ1 was first identified in a murine model of experimental asthma and an increase in its mRNA and protein levels were seen in a variety of T-helper (Th)2-mediated inflammatory settings [1], suggesting a role in the pathogenesis of Th2-mediated diseases. FIZZ1 protein administered into the lungs of mice has been shown to induce macrophage infiltration into the bronchoalveolar lavage (BAL) and collagen deposition in the lung $[2,3]$. Additional studies revealed that FIZZ1 protein has vasoconstrictive properties and is anti-apoptotic for lung fibroblasts [4, 5]. Although a biological effect of FIZZ1 has not been elucidated on intact tracheal smooth muscle (TSM), the strong induction of expression and these reports of pleiotropic biological activities led us to hypothesise that FIZZ1 may influence the functional behaviour of TSM. This could provide important clues to the role of FIZZ1 in such pulmonary diseases as asthma and, thus, may provide novel therapeutic opportunities [6-8].

In this study, increased levels of FIZZ1 mRNA and protein were demonstrated in trachea and/ or BAL from the ovalbumin (OA) model. Impairment of the epithelial layer was observed in rFIZZ1-treated trachea with an associated increased contractile response, over expression of myosin light chain kinase (MLCK), myosin light chain (MLC)-20 and activation of signalling molecules linked to mitogen-activated protein kinase (MAPK) pathway.

\section{MATERIALS AND METHODS}

\section{Preparation of animals and trachea}

Specific pathogen-free male BALB/C mice (5 week old) were housed at Pfizer Research (Cambridge, MA, USA) under pathogen-free conditions for the duration of the experiments. All studies were conducted in accordance with the National Institutes of Health Guide for the Care and Use of Laboratory Animals, as well as following guidelines from, and with the approval of, the Institutional Animal Care and Use Committee of Pfizer Research.

Animals were grouped as PBS-sensitised and -challenged (PBS/PBS), OA-sensitised and PBSchallenged (OA/PBS), and OA-sensitised and -challenged mice (OA/OA). Mice were injected intraperitoneally with PBS or OA $(20 \mu \mathrm{g})$ with $2.25 \mathrm{mg} \mathrm{Al}(\mathrm{OH})_{3}$ on day 0 and 14. From day 25 to 34 , mice were challenged with PBS or OA $(5 \%)$ for $30 \mathrm{~min} \cdot$ day $^{-1}$ for 10 consecutive days.

Tracheal rings from naïve mice were isolated and cultured overnight in DMEM in the absence and presence of 10 or $100 \mathrm{nM}$ rFIZZ1 (Leinco

\section{AFFILIATIONS}

Inflammation/Immunology, Pfizer Research, Cambridge, MA, USA.

CORRESPONDENCE

M.R. Bowman

Inflammation/lmmunology

Pfizer Research

200 Cambridge Park Drive Cambridge, MA 02140

USA

E-mail: michael.bowman@

pfizer.com

Received:

June 222009

Accepted after revision:

April 072010

First published online:

April 222010 
Technologies, St Louis, MO, USA) as well as $0.1 \mathrm{ng} \cdot \mathrm{mL}^{-1}$ lipopolysaccharide (LPS; $100 \mathrm{nM}$ rFIZZ1 contains an endotoxin level $<0.1 \mathrm{ng}$ ). In a separate study, the tension in fresh trachea and BAL cell counts were measured $24 \mathrm{~h}$ after the last treatment of mice receiving an intranasal PBS, $0.1 \mathrm{ng} \cdot \mathrm{mL}^{-1}$ LPS or $100 \mathrm{nM}$ rFIZZ1 (once a day for 5 days).

\section{Cell counts and protein preparation}

BAL from each sacrificed mouse was centrifuged (225 xg for $5 \mathrm{~min})$. The total BAL cells were counted and a sample applied to a glass slide, stained with Hema 3 Stain Set (Fisher Scientific, Pittsburgh, PA, USA), for the differential count of cells.

TSM tissue was collected by cutting away cartilage and physically scraping off the epithelium under a light microscope. The PBSand rFIZZ1-treated trachea and TSM were homogenised in lysis buffer containing $20 \mathrm{mM}$ MOSP, $2.0 \mathrm{mM}$ EGTA, $5.0 \mathrm{mM}$ EDTA, $30 \mathrm{mM}$ sodium fluoride, $40 \mathrm{mM} \beta$-glycerophosphate, $20 \mathrm{mM}$ sodium orthovanadate, $1.0 \mathrm{mM}$ phenylmethylsulfonyl-fluoride, $3.0 \mathrm{mM}$ benzamidine, $0.005 \mathrm{mM}$ pepstatin A, $0.01 \mathrm{mM}$ leupeptin and $0.5 \%$ Triton $\mathrm{X}-100$ at $\mathrm{pH} 7.2$. The supernatants were cleared by centrifugation and the protein concentrations measured by bicinchoninic acid assay.

\section{Examination of airway epithelium and epithelial cell culture and assay}

The status of the epithelial layer was examined in whole/ sectional trachea with and without $100 \mathrm{nM}$ rFIZZ1 or by mechanically removing the intraluminal surface by gently rubbing with polyethylene tubing (PE10) connected to a needle (30G1/2) followed by perfusion with $1.0 \mathrm{~mL}$ of air bubbles and then $1.0 \mathrm{~mL}$ Krebs-Henseleit solution [9]. The trachea was stained with Haematoxylin and eosin and was photographed using computer-based imaging under a light microscope.

Mouse tracheal epithelial cell (MTEC) culture was performed as previously described, with minor modification [10, 11]. Briefly, trachea was incubated in $1.5 \mathrm{mg} \cdot \mathrm{mL}^{-1}$ pronase for $18 \mathrm{~h}$ at $4{ }^{\circ} \mathrm{C}$. Cells were treated with $0.5 \mathrm{mg} \cdot \mathrm{mL}^{-1}$ crude pancreatic DNase I (Sigma-Aldrich, St Louis, MO, USA) on ice for $5 \mathrm{~min}$. After incubation in tissue culture plates for $3-4 \mathrm{~h}$ in $5 \% \mathrm{CO}_{2}$ at $37^{\circ} \mathrm{C}$, nonadherent cells were incubated in a plate coated with type I rat tail collagen (BD Biosciences, San Jose, CA, USA) in modified BEBM (Lonza, Walkersville, MD USA) containing $10 \mu \mathrm{g} \cdot \mathrm{mL}^{-1}$ insulin, $5 \mu \mathrm{g} \cdot \mathrm{mL}^{-1}$ transferrin, $25 \mathrm{ng} \cdot \mathrm{mL}^{-1}$ epidermal growth factor, $5 \mu \mathrm{g} \cdot \mathrm{mL}^{-1}$ adrenalin and $30 \mu \mathrm{g} \cdot \mathrm{mL}^{-1}$ bovine pituitary extract, $0.5 \mathrm{nM}$ hydrocortisone, $25 \mathrm{ng} \cdot \mathrm{mL}^{-1}$ human epidermal growth factor, $15 \mathrm{nM}$ triiodothyronine, $0.25 \mu \mathrm{g} \cdot \mathrm{mL}^{-1}$ gentamicin/amphotericin- $\mathrm{B}$ and $0.01 \mu \mathrm{M}$ retinoic acid in $5 \% \mathrm{CO}_{2}$ at $37^{\circ} \mathrm{C}$. MTEC were seeded on polycarbonate semi-permeable membrane $(0.4 \mu \mathrm{M}$ pore size; Corning Life Sciences, Lowell, MA, USA) and media was removed from upper chamber to establish an air-liquid interface, only lower chambers were provided with BEBM/DMEM $(1: 1, \mathrm{v} / \mathrm{v})$ containing $7.5 \mu \mathrm{L}$ retinoic acid and $750 \mu \mathrm{L}$ bovine serum albumin in the presence and absence of LPS and rFIZZ1.

Apoptotic MTEC death was examined in MTEC cultures $\left(5 \times 10^{4} \cdot \mathrm{mL}^{-1}\right)$ treated with PBS, $0.1 \mathrm{ng} \cdot \mathrm{mL}^{-1} \mathrm{LPS}$ or $100 \mathrm{nM}$ rFIZZ1 using Cell Death Detection ELISA ${ }^{\text {plus }}$ (Roche Applied
Science, Indianapolis, IN, USA) and calculated as an index of a fold change over a control.

Nitric oxide (NO) was examined by measuring an end product, [nitrite]. Briefly, aliquots $(50 \mu \mathrm{L})$ of supernatants from treated MTEC were mixed with $50 \mu \mathrm{L}$ Griess reagent (Bio-Rad, Hercules, CA, USA) at room temperature for $10 \mathrm{~min}$. The absorbance was read at $540 \mathrm{~nm}$ in an automated microplate reader [12].

\section{Pharmacodynamic studies}

The isometric tensions of TSM were examined as previously described [13]. Briefly, a change in the tension was recorded with a MP15 system (BIOPAC Systems, Inc., Goleta, CA, USA). A carbachol (CCh) concentration-response curve was completed in tracheal rings in the absence and presence of either rFIZZ1 or LPS. Concentration of the agonist was increased only after the force responses to the previous concentration had stabilised. In addition, a heat-inactivated $\mathrm{rFIZZ1}\left(70^{\circ} \mathrm{C} \times 60 \mathrm{~min}\right)$ was examined to verify its biological activity.

\section{Electrophoresis and quantification of proteins}

Aliquots of tracheal $\left(100 \mu \mathrm{g} \cdot \mathrm{well}^{-1}\right)$ and TSM $\left(10 \mu \mathrm{g} \cdot \mathrm{well}^{-1}\right)$ lysates were size-fractionated on a $4-20 \%$ SDS-PAGE gel and transferred to a nitrocellulose membrane and blocked with $5 \%$ nonfat dried milk in Tris-buffered saline. The membrane was individually incubated with primary antibodies to FIZZ1 (Antigenix America Inc., Huntington Station, NY, USA), MLCK, MLC-20, $\alpha$-actin, Gi $\alpha 1,2, \mathrm{Gq} \alpha 11, \beta$-actin (SigmaAldrich), G $\alpha 12 / 13$ (Santa Cruz Biotechnology, Inc., Santa Cruz, CA, USA), c-Raf, phospho-c-Raf, extracellular signalregulated kinase (ERK)1/2, phospho-ERK1/2, p38 MAPK and phospho-p38 MAPK (Cell Signaling, Inc., Danvers, MA, USA) at $4^{\circ} \mathrm{C}$ overnight and then incubated with horseradish peroxidase-conjugated secondary antibodies. Proteins were quantified using ImageJ and relative band intensity calculated as percentage of the intensity of the $\beta$-actin protein band.

\section{Gene chips}

Total RNA of trachea from treated mice was extracted and RNA was purified with Qiagen RNeasy minicolumns. This RNA was linearly amplified and biotin-labelled with Nugen's Ovation System (NuGEN Technologies, Inc., San Carlos, CA, USA) [14]. Approximately $1.5 \mu \mathrm{g}$ of purified and fragmented biotinylated CRNA, together with Wyeth standards for quantifying the amount of each transcript, was hybridised to the mouse gene chip array for $16-18 \mathrm{~h}$. Gene chips were scanned with an Agilent GeneArray scanner (Agilent, Santa Clara, CA, USA). The signals were normalised and quantified using Gene Logic MAS 5.0 software (Gene Logic, Gaithersburg, MD, USA).

\section{Data analysis}

At the end of an experiment, trachea were blotted on a gauze pad and weighed. Results were calculated as tension/TSM weight $\left(\mathrm{mg} \cdot \mathrm{mg}^{-1}\right)$ and expressed as a percentage of the mean value of the maximal responses in PBS-treated trachea.

All values were expressed as mean \pm SEM. Comparisons within groups of contractile agonist were performed by ANOVA. Unpaired t-tests were used to compare the effects of PBS, LPS and rFIZZ1. A p-value $<0.05$ was considered significant. 


\section{RESULTS}

\section{Features of the OA model}

The CCh-induced contraction was increased in trachea from the OA/OA-treated mice (fig. 1a). The difference in the force level was statistically significant when comparing either PBS/ PBS or OA/PBS versus OA/OA $(p<0.05, n=6)$. The cellular composition of the BAL was determined for the treated mice (fig. 1b-d). A large increase in the number of total BAL cells, lymphocytes and eosinophils was observed in the OA/OAtreated mice compared to those from the other two groups. $(\mathrm{p}<0.01, \mathrm{n}=6)$. The level of FIZZ1 mRNA expression in tracheal tissue was examined by transcriptional profiling and is shown in figure 2. The fold change in FIZZ1 mRNA expression in trachea from OA/OA-treated mice was increased 33-fold over that from either PBS/PBS- or OA/ PBS-treated mice. In contrast to the inability to measure FIZZ1 protein from either PBS/PBS- or OA/PBS-treated mice, the FIZZ1 protein was easily detected in the BAL and trachea from the OA/OA-treated mice (fig. 2). These data, together with those from previous publications, indicate that FIZZ1 is induced in Th2 inflammatory situations.

\section{Histological examination of airway epithelium}

There were no tissue oedema, unusual epithelial denudation and/or patchy shedding of epithelial cells on the luminal side
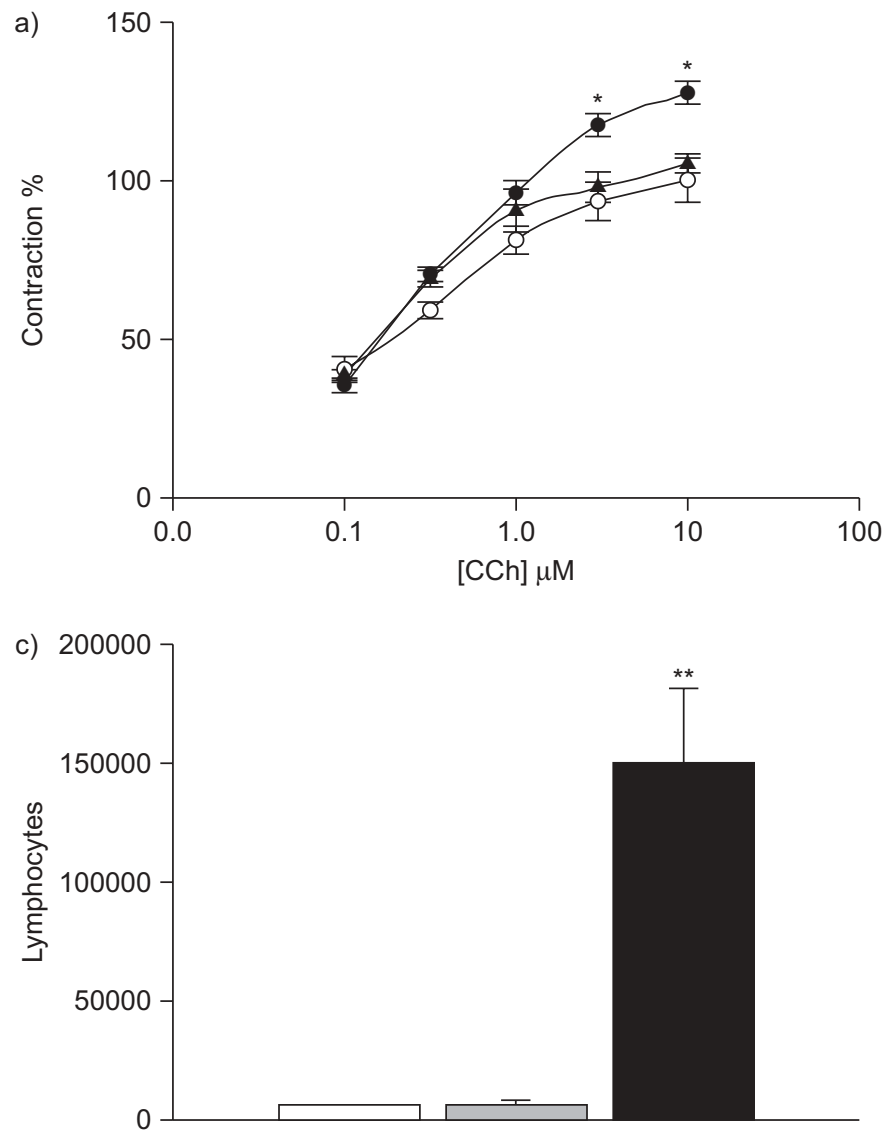

of the PBS/PBS-treated fresh trachea or the PBS-treated cultured trachea (fig. 3). In contrast, there was an obvious infiltration of inflammatory cells into epithelial layer/tracheal wall in OA/OA-exposed trachea (fig. 3a-c). In association with this infiltration, epithelial cells were destroyed with epithelial denudation or patchy shedding of the epithelial cells in the trachea. In rFIZZ1-treated cultured trachea, the epithelial layer was thinner and some of epithelium was denuded (fig. $3 \mathrm{~d}-\mathrm{f}$ ). However, the epithelial layer in fresh trachea from rFIZZ1exposed mice was intact with no epithelial denudation and inflammatory cells infiltrate in the trachea (fig. $3 g-i$ ). With the mechanical removal of epithelium, histological observation showed a similar state of epithelial denudation to that seen in rFIZZ1-treated trachea.

Effect of rFIZZ1, heat-inactivated FIZZ1 and LPS on trachea rFIZZ1-treated trachea induced a significantly increased force response (fig. $4 \mathrm{a}$ and c) compared to PBS-treated trachea $(\mathrm{p}<0.05, \mathrm{n}=6)$. Similarly, the increased expression of MLCK and MLC-20 was detected in rFIZZ1 treated TSM (fig. 4c). Heat-treated rFIZZ1 and LPS had no effect on the CChmediated force response (fig. $4 \mathrm{~d}$ ). There was a statistically significant difference detected in the force response between the native rFIZZ1 treated group and the other groups $(\mathrm{p}<0.05, \mathrm{n}=6)$.
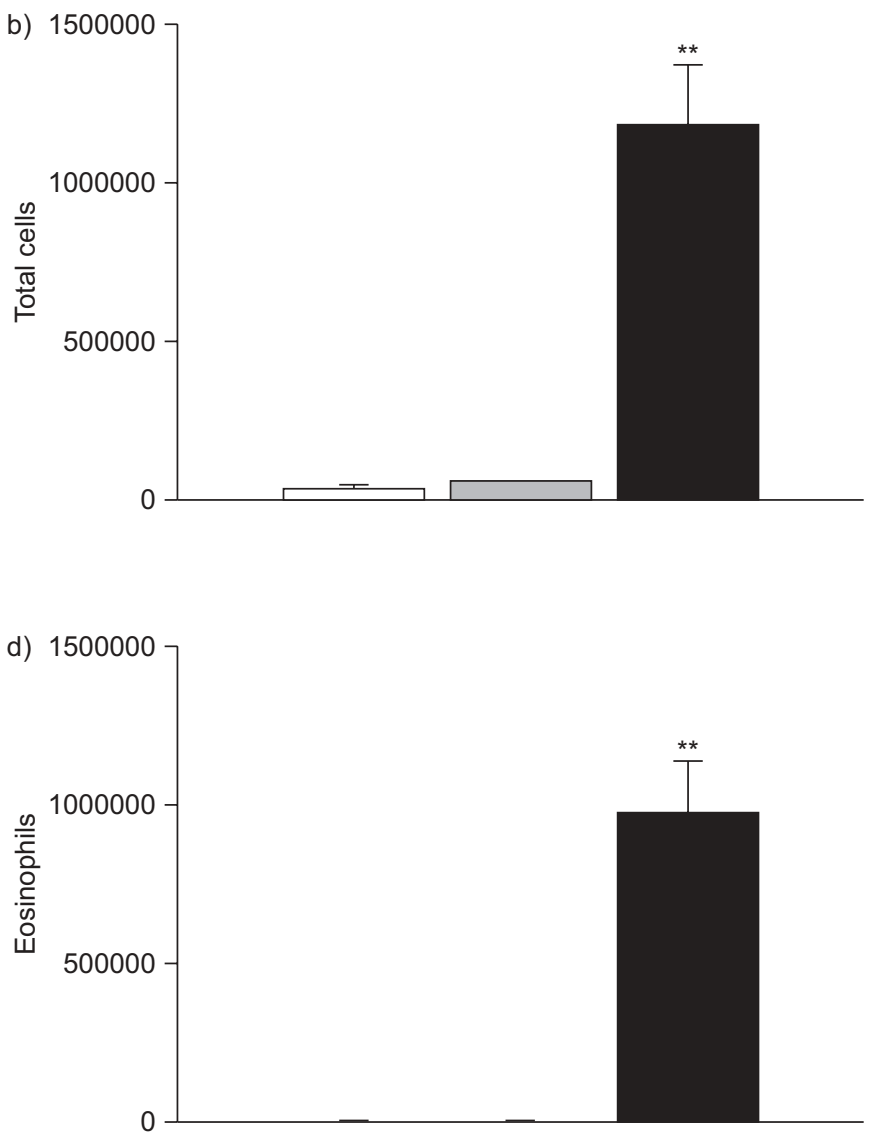

FIGURE 1. Tracheal smooth muscle contractility and the number of bronchoalveolar lavage (BAL) cells are enhanced in the ovalbumin (OA) model. a) Tracheal smooth muscle contractility and counts of b) total cells, c) lymphocytes and d) eosinophils from BAL were examined in PBS/PBS-treated mice ( $\square$ ), OA/PBS-treated mice ( $\square)$ and OA/ OA-treated mice ( $\mathbf{\square})$. O: PBS/PBS; $\mathbf{\Lambda}:$ OA/PBA; $\bullet$ : OA/OA. Data are presented as mean \pm sEM. CCh: carbachol. *: $p<0.05 ; * \star: p<0.01$ OA/OA-treated mice versus either PBS/PBS- or OA/PBS-treated mice. 
a)
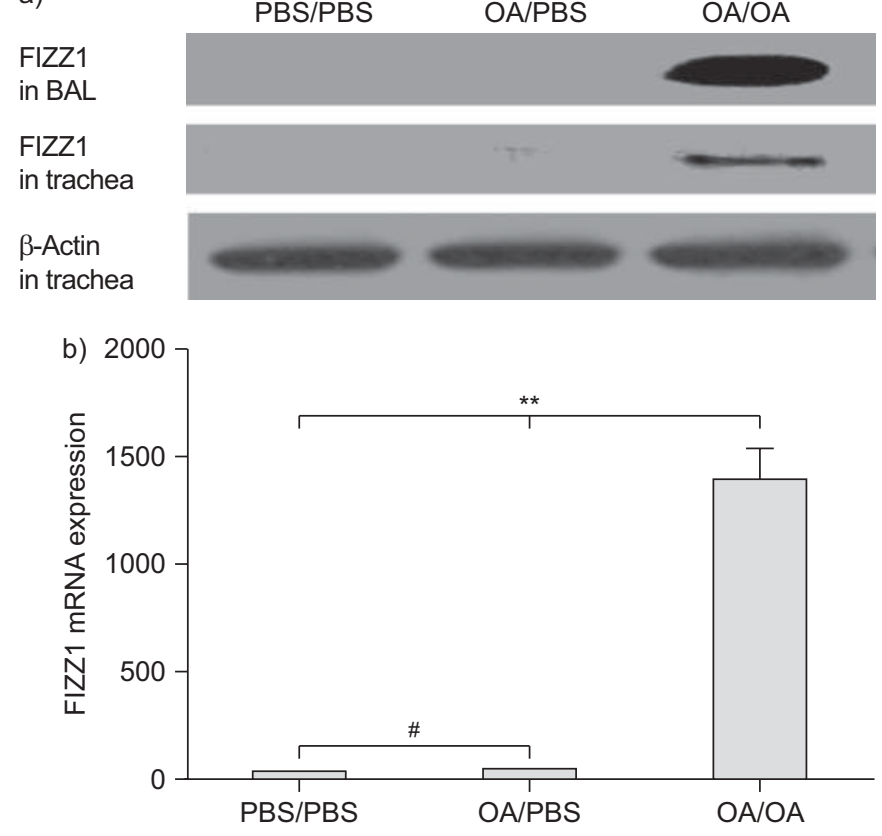

FIGURE 2. The signal of FIZZ1 mRNA and protein expression are increased in the ovalbumin (OA) model. a) The level of FIZZ1 protein in the bronchoalveolar lavage (BAL) and trachea from the treated mice was determined in reference to the level of $\beta$-actin in the tracheal sample. b) The FIZZ1 mRNA expression in trachea was assayed by transcriptional profiling. FIZZ1 levels are represented as the fold change of mRNA from trachea from mice treated with PBS/PBS, OA/PBS and OA/ OA versus trachea from naïve animals. Data are presented as mean \pm SEM. $* *: p<0.01 ; *: p=0.51$

\section{Force response of fresh trachea and BAL cell counts}

The BAL cell counts and tensions of fresh trachea were examined $24 \mathrm{~h}$ after the last treatment of mice receiving intranasal doses of PBS, LPS or rFIZZ1 (fig. 5). Significant increases in the CCh-evoked force response (fig. 5a) measured in the fresh trachea and in the number of BAL cells (fig. 5b) were detected in rFIZZ1-challenged mice compared to either PBS- or LPS-exposed mice $(\mathrm{p}<0.05, \mathrm{n}=5)$. In addition, a slight increase in the cell counts was observed in LPS-treated mice versus PBS-treated mice $(\mathrm{p}<0.05, \mathrm{n}=5)$.

\section{rFIZZ1 effects on MTEC and epithelium-denuded trachea}

In order to explore the mechanisms responsible for the FIZZ1mediated loss of tracheal epithelium, MTEC apoptosis was investigated. A significant increase $(\mathrm{p}<0.05$ or $\mathrm{p}<0.01 ; \mathrm{n}=3)$ in MTEC apoptosis was detected at all of the time-points after rFIZZ1 treatment compared to LPS treatment (fig. 6a). However, no induction of nitrite production was measured at any time-point (fig. 6b). With mechanical removal of the epithelium, there were significant differences in the force response between trachea with and without epithelium, as well as untreated and rFIZZ1-treated trachea without epithelium ( $p<0.05, n=8-19)$ (fig. 6c). Taken together, these data indicate that FIZZ1 cause epithelial cell death and this cell death, together with a direct effect on the smooth muscle, induces an increased tracheal contractile response.

\section{Protein expression in rFIZZ1-treated trachea}

Upon initiation of receptor-mediated signalling, the activation state of second messenger proteins is altered. We investigated the state of several $\mathrm{G}$ protein-coupled receptors as well as members of the c-Raf/MAPK/ERK pathways. The expression levels of $\alpha$ actin, Gi $\alpha 1,2$, Gq $\alpha 11$ and G $\alpha 12 / 13$ were similar between PBS- and rFIZZ1-treated trachea (fig. 7a). The expression of phosphorylated and unphosphorylated c-Raf, ERK1/2 and p38 MAPK showed a similar level of expression for most of the unphosphorylated proteins, whereas phospho-c-Raf, phospho-ERK1/2 and phospho-p38 MAPK in trachea and TSM tissues all statistically increased in expression after rFIZZ1 treatment $(\mathrm{p}<0.01$ or $\mathrm{p}<0.05$ versus PBS; $\mathrm{n}=3$ ) (fig. $7 \mathrm{~b}-\mathrm{d}$ ). These data indicate that correlated with an increase in TSM contraction, FIZZ1 induces an activation of the c-Raf/MAPK signalling pathway.

\section{DISCUSSION}

Several studies have tested the hypothesis that nonspecific airway hyperresponsiveness (AHR) in asthma is caused by increased force generation in the smooth muscle due to either increases in the size and number of individual muscle cells or a modification of the muscle's intracellular contractile signalling pathways [15-17]. In initial experiments, a mouse AHR model with a 10-day OA challenge was initially performed following a previously reported protocol modelling abnormal TSM function $[18,19]$. Our results show that this model is associated with a significant increase in CCh-evoked force and a large inflammatory infiltrate, mainly comprised of lymphocytes and eosinophils, into the BAL. In association with these findings, transcriptional profiling revealed that FIZZ1 mRNA expression in the trachea from OA/OA-treated mice was upregulated 30-fold over that from either PBS/PBS- or OA/PBS-treated animals. These data identified FIZZ1 as an inducible gene product within a process of local allergen-triggered airway inflammation. In support of this finding, FIZZ1 protein was detected in the BAL and trachea from OA/OA-treated mice, suggesting the particular importance of FIZZ1 as a proinflammatory mediator propagating allergic inflammation. It has been previously reported that FIZZ1 protein expression was significantly increased in the BAL from OA-treated mice, reaching levels as high as $5 \mu \mathrm{g} \cdot \mathrm{mL}^{-1}$ [1]. Due to the correlation of increased FIZZ1 protein expression and the induction of AHR in inflamed trachea, we postulated that FIZZ1 contributes to a cascade of effects culminating in TSM dysfunction.

Since FIZZ1 is found in airway epithelium [1, 4], it suggests that this protein exerts its effects in the local environment. In a direct rFIZZ1-treated ring we found the epithelial layer was significantly thinner and lacked histological intactness with epithelial denudation. This reveals that FIZZ1 acts on the airway epithelium and leads to a direct loss of the epithelial barrier similar to that seen in OA/OA-exposed trachea where an infiltration is associated with patchy epithelial layer destruction. Since epithelial damage is clinically associated with human asthma $[20,21]$ and often caused by a release of major basic proteins from infiltrating inflammatory cells into the inflamed airways [22, 23], our data suggest that the in vitro epithelial damage observed in the FIZZ1-exposed trachea is consistent with that of the in vivo asthmatic airway.

FIZZ1 was found to directly induce an increase in the CChgenerated force in TSM. In support of this result, expression levels 

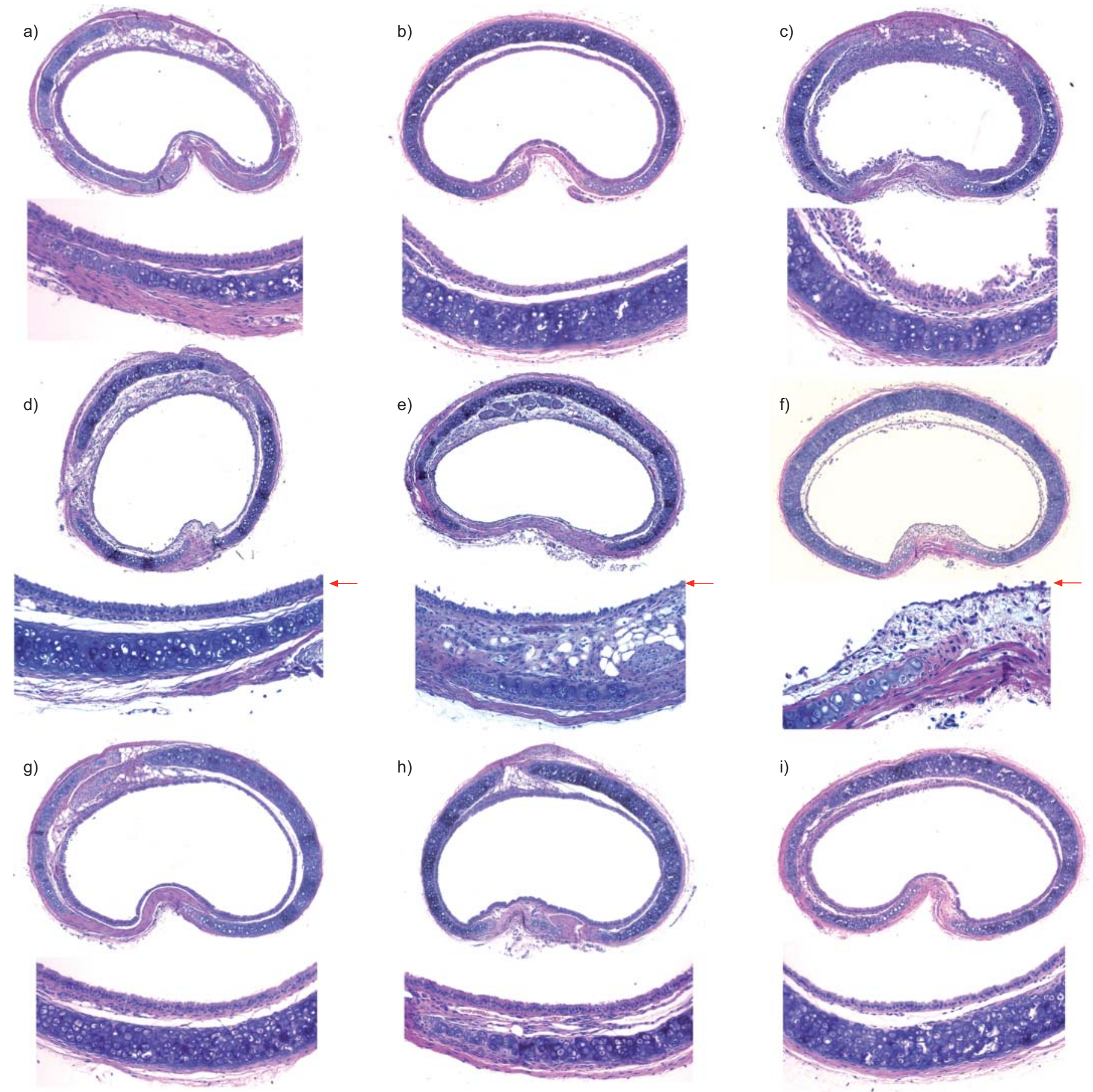

FIGURE 3. rFIZZ1 or mechanical removal results in the loss of the luminal epithelial layer. Histological examination of airway structure and the status of the airway epithelial layer were performed on frozen tracheae (either whole or sectional) from a-c) the ovalbumin (OA) model, $d-f$ ) $r F I Z Z 1$ cultured trachea and $g-i)$ fresh trachea from rFIZZ1-treated mice. a) Trachea from mice sensitised and challenged with PBS (PBS/PBS), b) trachea from PBS/OA mice, c) trachea from OA/OA mice, d) trachea cultured in PBS, e) trachea cultured in rFIZZ1, f) trachea with epithelial cell layer mechanically removed, g) fresh trachea from PBS-treated mice, h) fresh trachea from lipopolysaccharidetreated mice and i) fresh trachea from rFIZZ1-treated mice. All sections were viewed by light microscopy at magnifications of $\times 4.0$ and $\times 20$.

of MLCK and its primary substrate, MLC-20, were significantly increased in the TSM. This finding provides an important molecular basis to fully understand the force development observed in the FIZZ1-treated trachea and supports the conclusion that FIZZ1 alters the contractile property of the TSM by influencing the expression level of contractile proteins within the tissue. It is well documented that a key event in the regulation of TSM contraction is the phosphorylation/dephosphorylation of the regulatory light chain of myosin catalysed by the calmodulinactivated MLCK [24-26]. An exposure to inflammatory mediators induces smooth muscle dysfunction with an increase in MLCK/ MLC-20 expression [27], suggesting that the TSM contractile 
a)

CCh $0.030 .10 .3 \quad 1.0 \quad 3.010$

PBS

rFIZZ1 10

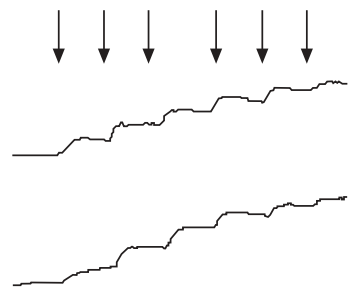

$0.75 \mathrm{~g}$

$3 \mathrm{~min}$

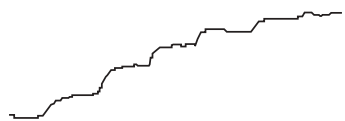

b)

PBS

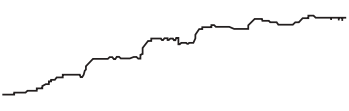

LPS $0.1 \mathrm{ng} \cdot \mathrm{mL}^{-1}$

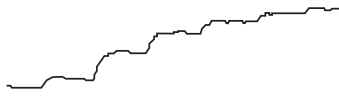

$0.75 \mathrm{~g}$

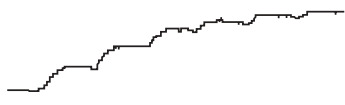

Heat-rFIZZ1 100

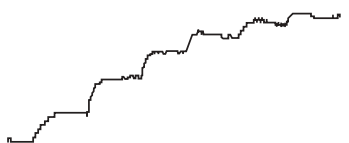

rFIZZ1 100 d)

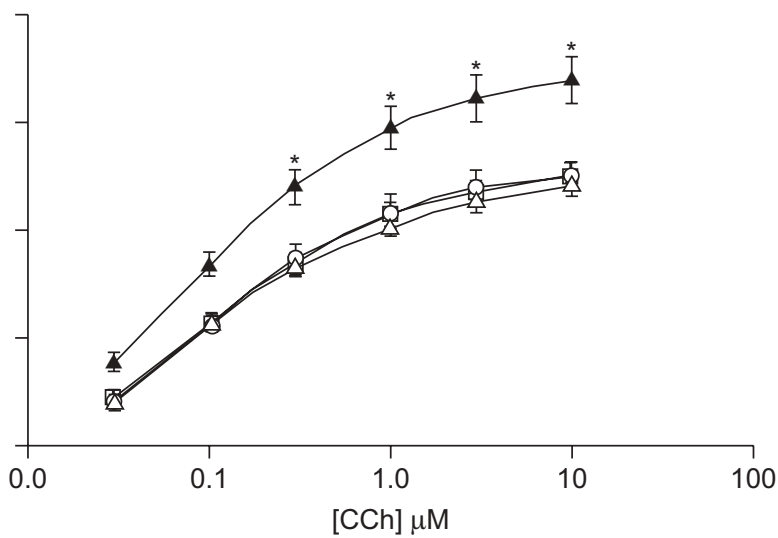

FIGURE 4. rFIZZ1 increases carbachol (CCh)-generated tracheal smooth muscle (TSM) force. a, b) The CCh-generated force in PBS-treated trachea, lipopolysaccharide (LPS)-treated trachea and rFIZZ1-treated trachea was recorded as original tracings. c, d) Cumulative concentration-response curves of isometric tension to CCh stimulation were completed in PBS- and native rFIZZ1-treated trachea (10 or 100 nM). Myosin light chain kinase (MLCK) and myosin light chain-20 (MLC-20) protein expression levels in TSM were measured in relation to the expression level of $\beta$-actin in the same tissue (c; insert). b, d) The CCh dose-response curves were also performed in trachea treated with $0.1 \mathrm{ng} \cdot \mathrm{mL}^{-1}$ of LPS, $100 \mathrm{nM}$ native rFIZZ1 and $100 \mathrm{nM}$ heat-inactivated rFIZZ1. $\mathbf{a}$ : rFIZZ1 $100 \mathrm{nM}$; $:$ rFIZZ1 $10 \mathrm{nM} ; 0$ : PBS; $\mathbf{\Lambda}: \mathrm{rFIZZ1;} \square:$ Heat-rFIZZ1; $\triangle$ : LPS. Data are presented as mean \pm SEM. ${ }^{*}: \mathrm{p}<0.05 \mathrm{rFIZZ1}$ versus PBS or heat-treated rFIZZ1 groups.

apparatus may be surrounded and bombarded by inflammatory mediators. Due to the histological finding that culturing with FIZZ1 resulted in epithelial denudation, there was a concern that the increased force response was a consequence of the epithelial damage. Because there is no known intrinsic linkage in the contractile mechanism between epithelial damage and the

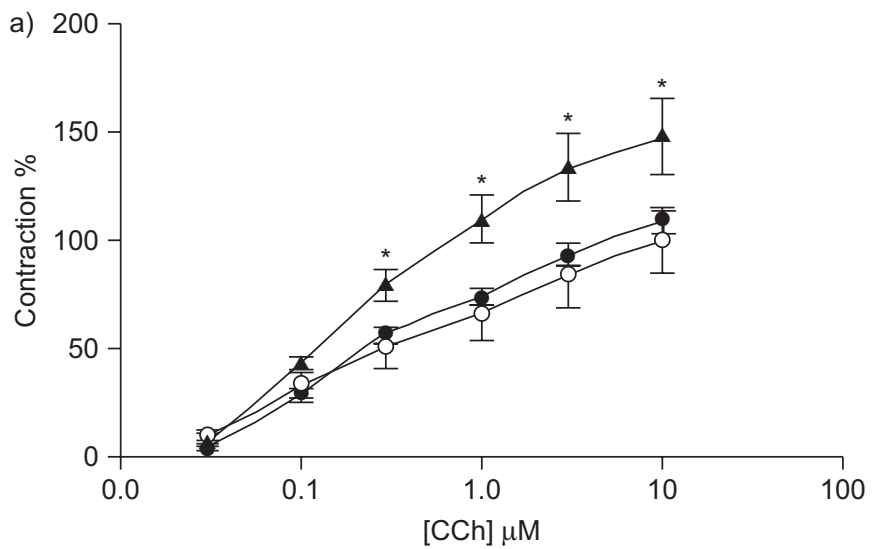

activation of MLCK, the FIZZ1 effect on CCh-elicited contraction should be considered a dual entity of two separate effects, one on the epithelium and another on the TSM. In support of the observed effect of FIZZ1 protein on cultured trachea, mice given an in vivo administration of rFIZZ1 protein showed significant increases in both the force response of freshly isolated trachea and

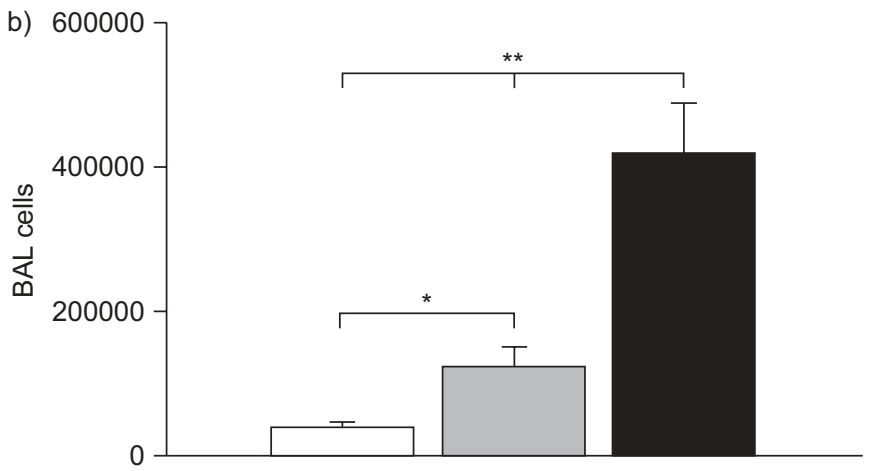

FIGURE 5. In vivo administration of rFIZZ1 enhances the contractile response of fresh trachea and increases the bronchoalveolar lavage (BAL) cell count. a) Force response in freshly isolated trachea and b) counts of BAL cells were examined in mice receiving intranasal PBS $(\bigcirc$ and $\square), 0.1 \mathrm{ng} \cdot \mathrm{mL}^{-1}$ lipopolysaccharide (LPS; $\bullet$ and $\left.\square\right)$ or $100 \mathrm{nM} \mathrm{rFIZZ1} \mathrm{(once} \mathrm{a} \mathrm{day} \mathrm{for} 5$ days; $\boldsymbol{\Lambda}$ and $\mathbf{\square}$ ). Data are presented as mean \pm SEM. CCh: carbachol. *: $\mathrm{p}<0.05$; ${ }^{*}$ : $\mathrm{p}<0.01$ versus either PBS- or LPS-treated mice. 

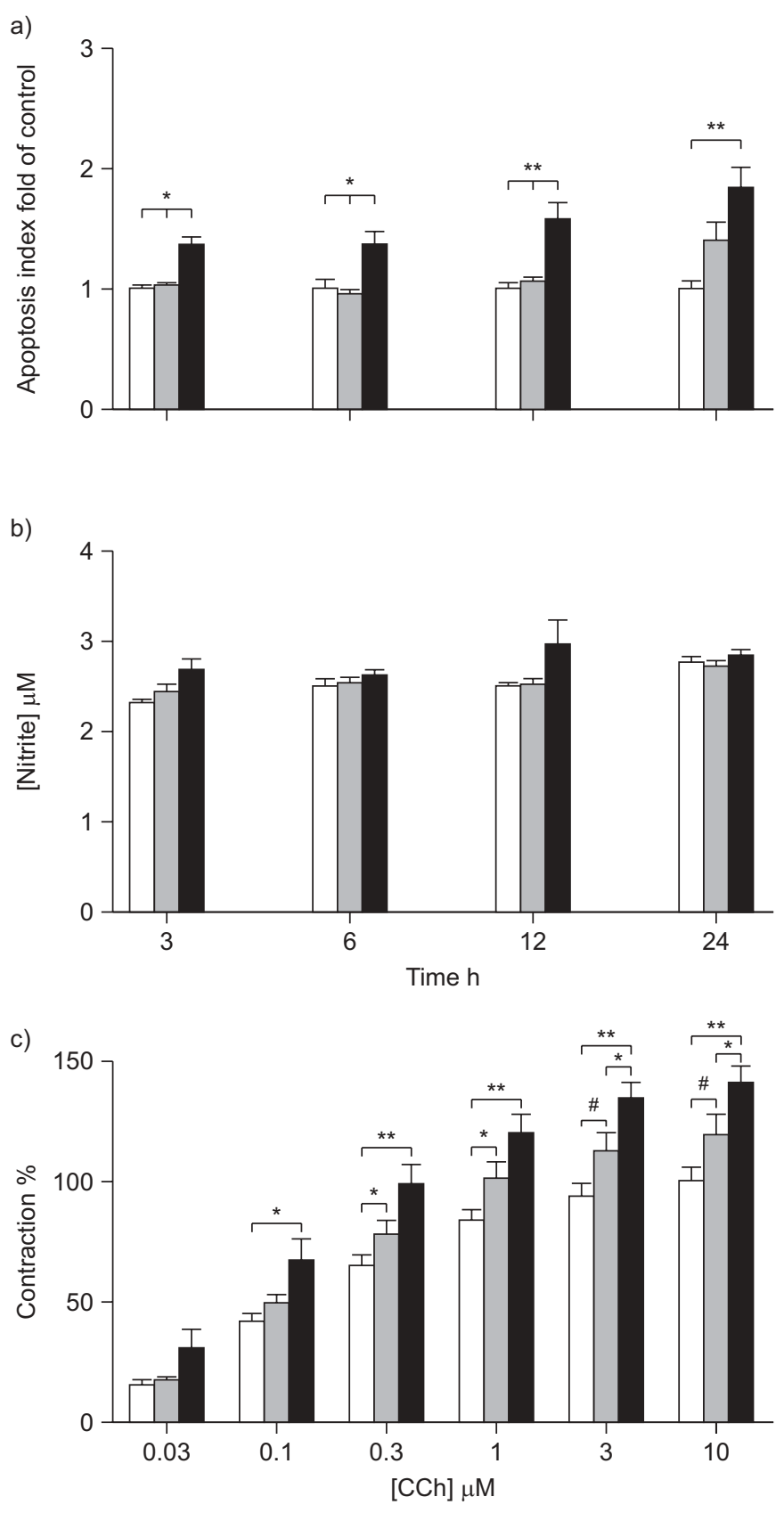

FIGURE 6. The effect of $\mathrm{rFIZZ1}$ on mouse tracheal epithelial cell culture (MTEC) and trachea without intact epithelium. a) MTEC apoptosis index and b) [Nitrite] were examined in supernatants from treated MTEC. $\square$ : PBS; 口: lipopolysaccharide; $\mathbf{\square}$ : rFIZZ1. c) Cumulative dose-response curves of isometric tension to carbachol (CCh) stimulation were measured in trachea with epithelium $(\square)$ and those with mechanically removed epithelium (EP-; $\square$ ) treated with PBS or FIZZ1. a : EP-/FIZZ1. All tension measurements for the groups $(n=8-19)$ are expressed as mean \pm SEM. ${ }^{*}: p<0.05$; ${ }^{* *}: p<0.01 ;{ }^{*}: p<0.07$.

in the number of BAL cells compared to either PBS- or LPStreated animals. Because the epithelial layer of freshly isolated trachea from FIZZ1-treated mice was intact with no cellular infiltrate, it leads us to conclude that FIZZ1 protein participates in modulating lung inflammation and the increased force activity in the trachea is due to a dysfunction of TSM rather than an influx of inflammatory cells.
Due to the histological changes in epithelial layer observed in rFIZZ1-treated trachea, the apoptosis of MTEC was determined within the time-period of the study. The results show a significant increase in apoptosis, indicating that FIZZ1 acts directly on airway tissue enhancing epithelial cell death. Further investigation showed no change in [nitrite] throughout this time period, suggesting a loss of NO was not responsible for the increased force response. In order to clarify whether or not the epithelial damage contributes to the increased force response in FIZZ1-exposed trachea, epithelial cells were mechanically removed from trachea of naïve mice. Our results show an increased force response in the epithelium-denuded trachea, demonstrating the importance of the epithelial barrier in the protection of TSM from direct exposure to contractile agonists. Since the epithelium-denuded trachea treated with rFIZZ1 showed an increased force level as compared to that of denuded trachea without rFIZZ1 treatment, it is reasonable to conclude that this protein exerts separable effects involving both the epithelium and TSM, representing different stages in the process of abnormal smooth muscle force development.

There has been growing awareness that pro-inflammatory proteins are able to modulate the functional properties of TSM [13]. To investigate the molecular mechanism(s) responsible for the FIZZ1-induced changes in the TSM functional response, the expression level of smooth muscle $\alpha$-actin was assessed in FIZZ-1-treated trachea based on a previous report that the transfection of a FIZZ1-expressing plasmid into lung fibroblasts stimulated $\alpha$-actin production and induced vasoconstrictive properties $[3,4]$. Our results showed that $\alpha$-actin was expressed at a similar level in both rFIZZ1- and PBStreated tissues, indicating that FIZZ1 did not exert its effects by changing the expression of this contractile element. Since the muscarinic receptor preferentially couples to G-proteins leading to an increase in MLC-20 phosphorylation and the associated muscle contraction, it is possible that the FIZZ1 effect is mediated through the activation of Gs $\alpha, \mathrm{Gi} \alpha, \mathrm{Gq} \alpha$ or G $\alpha 12 / 13$, amplifying the receptor-mediated generation of second messengers [28]. Our results show that the expression of the G-proteins in rFIZZ1-treated trachea was identical to the level detected in PBS-treated trachea, indicating that changes in the expression of these G-proteins is not involved in the event.

Upon binding their cognate ligand, receptor tyrosine kinases (RTK) in the plasma membrane activate $\mathrm{Ca}^{2+}$ mobilisation, inducing TSM force generation, through a pathway distinct from that used by the G-protein-coupled receptors [29, 30]. The activation of RTKs may produce a downstream effect contributing to the activation of c-Raf-mediated MAPK signal transduction pathways eventually leading to an increase in MLC-20 phosphorylation [31-33]. A recent study suggested that ERK1/2 and p38MAPK in fibroblasts were activated by FIZZ1 and an inhibitor of the MAPK pathway suppressed this activation [5]. Our results show that FIZZ1 treatment induces high levels of phospho-c-Raf, phospho-ERK1/2 and phosphop38 MAPK not only in tracheal rings but also in TSM tissue, indicating that FIZZ1 is sufficient to cause the activation of this arm of the MAPK signalling pathway and it directly acts on the TSM tissue. The lack of change in the expression levels of $G$ proteins and the increased c-Raf/MAPK and MLC-20 phosphorylation, leads us to conclude that FIZZ1 regulation of the 
a)
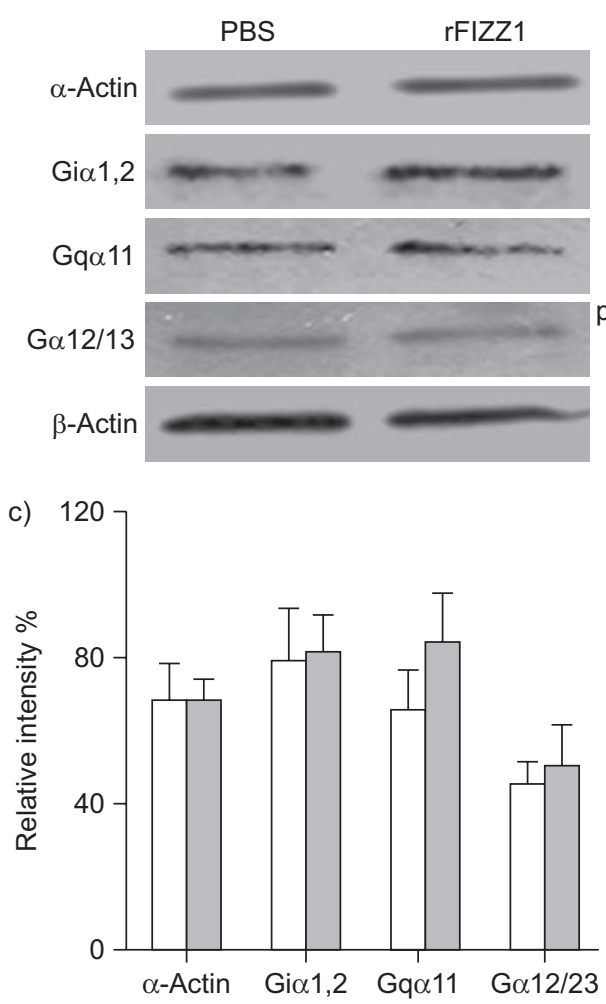

b)

\begin{tabular}{cc}
\cline { 2 - 2 }$\quad \mathrm{rFIZZ1}$ \\
$\mathrm{c}$-Raf
\end{tabular}

Trachea

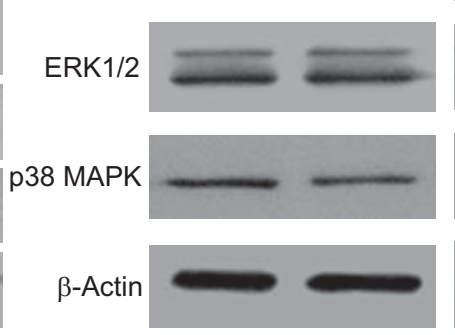

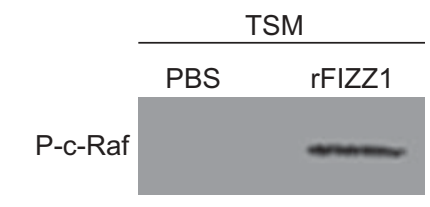

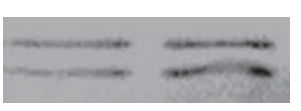

P-ERK1/2
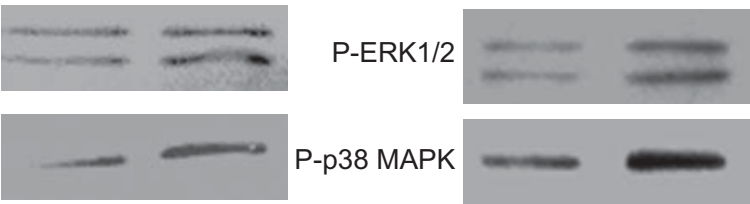

P-p38 MAPK

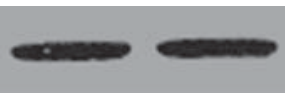

$\beta$-Actin

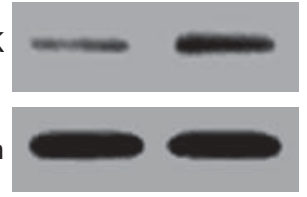

d) 80

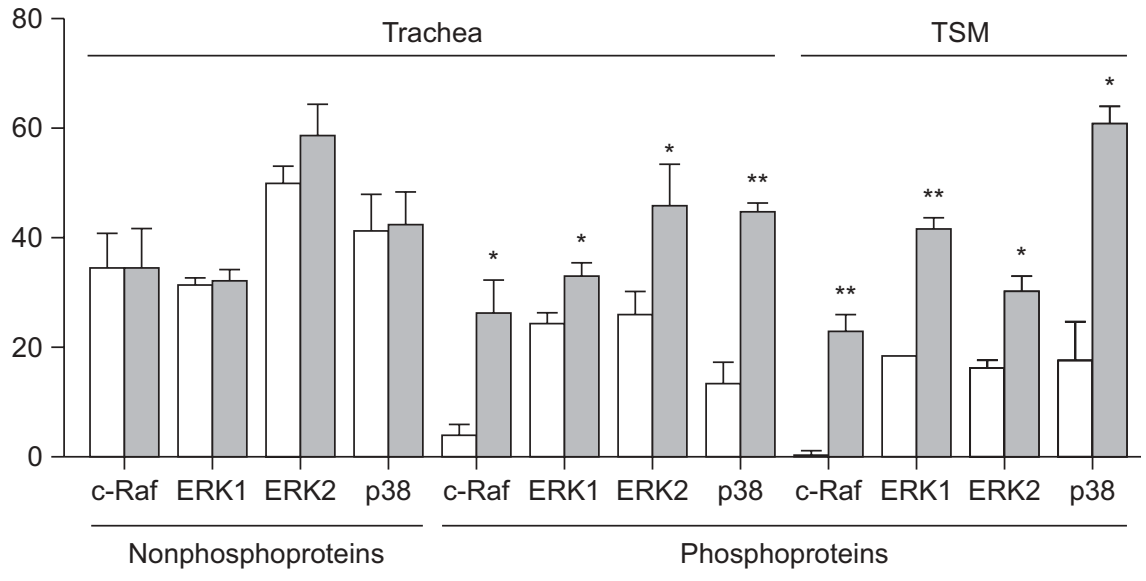

FIGURE 7. Phosphorylation of c-Raf, extracelluar signal-regulated kinase (ERK) 1/2 and p38 mitogen-activated protein kinase (MAPK) is increased in rFIZZ1-treated trachea. a) The expression levels of $\alpha$-actin and various G proteins, as well as proteins involved in the MAPK pathway such as b) c-Raf, phospho (P)-c-Raf, ERK1/2, P-ERK1/2, p38 MAPK and P-p38 MAPK were examined in either $100 \mathrm{nM} \mathrm{rFIZZ1-} \mathrm{or} \mathrm{PBS-treated} \mathrm{trachea} \mathrm{or} \mathrm{tracheal} \mathrm{smooth} \mathrm{muscle} \mathrm{(TSM).} \mathrm{c,} \mathrm{d)} \mathrm{Quantification} \mathrm{of} \mathrm{the} \mathrm{intensity} \mathrm{of} \mathrm{the}$ protein bands was performed using ImageJ. $\square$ : PBS; 1 ; rFIZZ1. *: $p<0.05$; **: $p<0.01$ versus PBS $(n=3)$.

CCh-evoked force appears to act through a c-Raf-linked MAPK signalling cascade leading to an increase in MLC-20 phosphorylation and enhanced TSM contraction.

In conclusion, our results indicate that FIZZ1 enhances the TSM contractile response with an associated increase in MLCK and MLC-20 expression levels. The increased force generation observed in FIZZ1-treated trachea is caused by an impairment of the airway epithelium and an activation of a c-Raf-ERK1/2p38 MAPK signalling pathway in the contracting TSM.

\section{STATEMENT OF INTEREST}

Statements of interest for all authors can be found at www.erj. ersjournals.com/site/misc/statements.xhtml

\section{REFERENCES}

1 Holcomb IN, Kabakoff RC, Chan B, et al. FIZZ1, a novel cysteinerich secreted protein associated with pulmonary inflammation, defines a new gene family. EMBO J 2000; 19: 4046-4055.

2 Liu $\mathrm{T}$, Jin $\mathrm{H}$, Ullenbruch $\mathrm{M}$, et al. Regulation of found in inflammatory zone 1 expression in bleomycin-induced lung fibrosis: role of IL-4/IL-13 and mediation via STAT-6. J Immunol 2004; 173: 3425-3431.

3 Liu T, Dhanasekaran SM, Jin H, et al. FIZZ1 stimulation of myofibroblast differentiation. Am J Pathol 2004; 164: 1315-1326.
4 Teng X, Li D, Champion HC, et al. FIZZ1/RELM $\alpha$, a novel hypoxia-induced mitogenic factor in lung with vasoconstrictive and angiogenic properties. Circ Res 2003; 92: 1065-1067.

5 Chung MJ, Liu T, Ullenbruch M, et al. Antiapoptotic effect of found in inflammatory zone (FIZZ) 1 on mouse lung fibroblasts. J Pathol 2007; 212: 180-187.

6 Oliver BG, Black JL. Airway smooth muscle and asthma. Allergol Int 2006; 55: 215-223.

7 Halayko AJ, Tran T, Ji SY, et al. Airway smooth muscle phenotype and function: interactions with current asthma therapies. Curr Drug Targets 2006; 7: 525-540.

8 Solway J, Irvin CG. Airway smooth muscle as a target for asthma therapy. N Engl J Med 2007; 356: 1367-1369.

9 Liu JQ, Yang D, Folz RJ. A novel bronchial ring bioassay for the evaluation of small airway smooth muscle function in mice. Am J Physiol Lung Cell Mol Physiol 2006; 291: L281-L288.

10 You Y, Richer EJ, Huang T, et al. Growth and differentiation of mouse tracheal epithelial cells: selection of a proliferative population. Am J Physiol Lung Cell Mol Physiol 2002; 283: L1315-L1321.

11 Mathew B, Park GY, Cao H, et al. Inhibitory kB kinase 2 activates airway epithelial cells to stimulate bone marrow macrophages. Am J Respir Cell Mol Biol Physiol 2007; 36: 562-572.

12 Chen H, MacLeod C, Deng B, et al. CAT-2 amplifies the agonistevoked force of airway smooth muscle by enhancing sperminemediated phosphatidylinositol-(4)-phosphate-5-kinase- $\gamma$ activity. Am J Physiol Lung Cell Mol Physiol 2007; 293: L883-L891.

13 Chen $\mathrm{H}$, Tliba O, Besien CV, et al. Airway hyper-responsiveness: from molecules to bedside selected contribution: TNF- $\alpha$ modulates 
murine tracheal rings responsiveness to G-protein-coupled receptor agonists and KCl. J Appl Physiol 2003; 95: 864-872.

14 Kurn N, Chen P, Heath DJ, et al. Novel isothermal, linear nucleic acid amplification systems for highly multiplexed applications. Clin Chem 2005; 51: 1973-1981.

15 Wiggs BR, Moreno R, Hogg JC, et al. A model of the mechanics of airway narrowing. J Appl Physiol 1990; 69: 849-860.

16 Chiba Y, Sakai H, Misawa M. Augmented acetylcholine-induced translocation of RhoA in bronchial smooth muscle from antigeninduced airway hyperresponsive rats. Br J Pharmacol 2001; 133: 886-890.

17 Martin JG, Duguet A, Eidelman DH. The contribution of airway smooth muscle to airway narrowing and airway hyperresponsiveness in disease. Eur Respir J 2000; 16: 349-354.

18 Matsubara S, Li G, Takeda K, et al. Inhibition of spleen tyrosine kinase prevents mast cell activation and airway hyperresponsiveness. Am J Respir Crit Care Med 2006; 173: 56-63.

19 Taube C, Wei X, Swasey CH, et al. Mast cells, FcRI, and IL-13 are required for development of airway hyperresponsiveness after aerosolized allergen exposure in the absence of adjuvant. J Immunol 2004; 172: 6398-6406.

20 Cokugras H, Akcakaya N, Seckin I, et al. Ultrastructural examination of bronchial biopsy specimens from children with moderate asthma. Thorax 2001; 56: 25-29.

21 White SR, Dorscheid DR. Corticosteroid-induced apoptosis of airway epithelium a potential mechanism for chronic airway epithelial damage in asthma. Chest 2002; 122: 2785-2845.

22 Flavahan NA, Slifman NR, Gleich GJ, et al. Human eosinophil basic protein causes hyperreactivity of respiratory smooth muscle. Am Rev Respir Dis 1988; 138: 685-688.
23 Gundel RH, Letts LG, Gleich GJ. Human eosinophil major basic protein induces airway contraction and airway hyperresponsiveness in primates. J Clin Invest 1991; 87: 1470-1473.

24 Somlyo AP, Somlyo AV. Signal transduction and regulation in smooth muscle. Nature 1994; 372: 231-236.

25 Kamm KE, Stull JT. Dedicated myosin light chain kinases with diverse cellular functions. J Biol Chem 2001; 276: 4527-4530.

26 Jiang H, Rao K, Halayko AJ, et al. Ragweed sensitization-induced increase of myosin light chain kinase content in canine airway smooth muscle. Am J Respir Cell Mol Biol 1992; 7: 567-573.

27 Fernandes DJ, Mitchell RW, Lakser O, et al. Invited review: do inflammatory mediators influence the contribution of airway smooth muscle contraction to airway hyperresponsiveness in asthma? J Appl Physiol 2003; 95: 844-853.

28 Schramm CM, Grunstein MM. Assessment of signal transduction mechanisms regulating airway smooth muscle contractility. Am J Physiol Lung Cell Mol Physiol 1992; 262: L119-L139.

29 Hubbard SR, Miller WT. Receptor tyrosine kinases: mechanisms of activation and signaling. Curr Opin Cell Biol 2007; 19: 117-123.

30 Berlin AA, Hogaboam CM, Lukacs NW. Inhibition of SCF attenuates peribronchial remodeling in chronic cockroach allergen-induced asthma. Lab Invest 2006; 86: 557-565.

31 McKay MM, Morrison DK. Integrating signals from RTKs to ERK/ MAPK. Oncogene 2007; 26: 3113-3121.

32 Klingenberg D, Gündüz D, Härtel F, et al. MEK/MAPK as a signaling element in ATP control of endothelial myosin light chain. Am J Physiol Cell Physiol 2004; 286: C807-C812.

33 Klemke RL, Cai S, Giannini AL, et al. Regulation of cell motility by mitogen-activated protein kinase. J Cell Biol 1997; 137: 481-492. 